\title{
Classification des formes quadratiques réelles : un contre-exemple à la finitude
}

\author{
par
}

\section{David-Olivier Jaquet-Chiffelle et François Sigrist (Neuchâtel)}

1. Introduction. On doit à G. Voronoï [Vo] un algorithme de classification complète des formes quadratiques parfaites. Il est dès lors possible, en principe, de déterminer en un temps fini la constante d'Hermite $\gamma_{n}$, qui décrit dans $\mathbb{R}^{n}$ la densité maximale des empilements de sphères en réseau.

L'énorme complexité de l'algorithme lui donne une limite naturelle : il semble actuellement impensable de dépasser la dimension 8 , où les explorations ont déjà fourni des milliers de formes parfaites. Signalons cependant que la constante $\gamma_{8}$ a été trouvée par une approche différente (Blichfeldt 1926), et que sa valeur vient d'être confirmée par la détermination de $\gamma_{7}$ [Ja1].

Dans [BMS], on envisage la restriction de l'algorithme de Voronoï à un sous-espace affine $T$ de l'espace vectoriel des formes quadratiques réelles (il faut introduire une restriction géométrique, qui sera clairement remplie cidessous : les empilements de sphères associés aux formes $T$-parfaites doivent être connexes). Dans une telle situation, l'algorithme est exhaustif, mais l'existence d'une condition d'arrêt n'est pas établie. Un des exemples ciaprès montrera qu'elle n'existe pas en général. Mentionnons cependant un résultat important [Ja2] : Dans le cas des $G$-formes (invariantes sous l'action d'un groupe fini $G$ ), il n'existe qu'un nombre fini de formes $G$-parfaites, à $G$-équivalence près.

2. Fonctionnement de l'algorithme de Voronoï en dimension 2. A $G l(2, \mathbb{Z})$-équivalence près, il n'y a qu'une seule forme parfaite à deux variables : $x^{2}-x y+y^{2}$. Celle-ci a les trois paires de vecteurs minimaux $\pm\left(\begin{array}{l}1 \\ 0\end{array}\right)$, $\pm\left(\begin{array}{l}0 \\ 1\end{array}\right)$, et $\pm\left(\begin{array}{l}1 \\ 1\end{array}\right)$. Comme les formes parfaites sont caractérisées par leurs vecteurs minimaux, on déduit la liste complète des formes parfaites en dimension 2 : elles ont les vecteurs minimaux $\pm \vec{u}= \pm\left(\begin{array}{l}u_{1} \\ u_{2}\end{array}\right), \pm \vec{v}= \pm\left(\begin{array}{l}v_{1} \\ v_{2}\end{array}\right)$, et $\pm(\vec{u}+\vec{v})$ ou $\pm(\vec{u}-\vec{v})$. Il est nécessaire et suffisant que $\left|u_{1} v_{2}-u_{2} v_{1}\right|=1$ pour obtenir une forme parfaite. C'est donc à l'aide des fractions de Farey 
que l'on décrit les formes, et par ailleurs leurs relations de contiguïté. En effet, la forme de vecteurs minimaux $\pm \vec{u}, \pm \vec{v}, \pm(\vec{u}+\vec{v})$ a trois formes qui lui sont contiguës, ayant pour vecteurs minimaux respectifs $\{ \pm \vec{u}, \pm \vec{v}, \pm(\vec{u}-\vec{v})\}$, $\{ \pm \vec{u}, \pm(2 \vec{u}+\vec{v}), \pm(\vec{u}+\vec{v})\},\{ \pm(\vec{u}+2 \vec{v}), \pm \vec{v}, \pm(\vec{u}+\vec{v})\}$. Une illustration parlante consiste à représenter les voisines de l'ellipse $x^{2}-x y+y^{2}=1$ qui sont $x^{2}+x y+y^{2}=1, x^{2}-3 x y+3 y^{2}=1$ et $3 x^{2}-3 x y+y^{2}=1$. Le passage d'une ellipse à sa voisine consiste à suivre le faisceau à un paramètre qui les relie (une ellipse et sa voisine ont quatre points communs) : ceci est précisément le mécanisme général de l'algorithme de Voronoï.

3. Etude d'un sous-espace linéaire à l'aide de l'algorithme de Voronoï relatif. Prenons le sous-espace $T$ de l'ensemble des formes quadratiques à deux variables décrit par le nombre réel $\phi>0$ : on exige que la droite de pente $\phi$ soit une direction propre de la matrice $\left(\begin{array}{ll}a & b \\ b & c\end{array}\right)$ décrivant la forme $a x^{2}+2 b x y+c y^{2}$. Pour les vecteurs minimaux d'une telle forme, on a le

Lemme. Supposons que la forme $a x^{2}+2 b x y+c y^{2}$ appartienne à T. Alors

(i) Si $\vec{u}=\left(\begin{array}{l}u_{1} \\ u_{2}\end{array}\right)$ est un vecteur minimal, alors la fraction $\left|u_{2} / u_{1}\right|$ est un segment initial du développement de $\phi$ en fraction continue.

(ii) Si $\vec{u}=\left(\begin{array}{l}u_{1} \\ u_{2}\end{array}\right)$ et $\vec{v}=\left(\begin{array}{l}v_{1} \\ v_{2}\end{array}\right)$ sont deux vecteurs minimaux indépendants, alors $\left|u_{1} v_{2}-u_{2} v_{1}\right|=1$.

Preuve. (i) Si $u_{2} / u_{1}$ n'est pas une approximation de $\phi$, il existe $w_{1}$ et $w_{2}$ tels que $\left|w_{1} \phi-w_{2}\right| \leq\left|u_{1} \phi-u_{2}\right|$ et $w_{1} \leq u_{1}$. Le point de coordonnées entières $\left(w_{1}, w_{2}\right)$ est intérieur à l'ellipse, et le vecteur $\vec{u}$ n'est donc pas minimal.

(ii) Le parallélogramme construit sur les extrémités de $\pm \vec{u}$ et $\pm \vec{v}$ a l'aire $2\left|u_{1} v_{2}-u_{2} v_{1}\right|$. Mais la valeur de la constante d'Hermite $\gamma_{2}=2 / \sqrt{3}$ implique que l'aire de l'ellipse ne peut pas dépasser $2 \pi / \sqrt{3}=3.62 \ldots$

Si l'on pose $\kappa=\phi-1 / \phi$, un calcul facile montre que $T$ est de dimension 2 , et qu'on peut prendre la base

$$
T_{1}=\left(\begin{array}{cc}
1 & -1 / \kappa \\
-1 / \kappa & 0
\end{array}\right), \quad T_{2}=\left(\begin{array}{cc}
0 & 1 / \kappa \\
1 / \kappa & 1
\end{array}\right) .
$$

La matrice-unité $Q(0)=T_{1}+T_{2}$ est $T$-parfaite, puisqu'elle a deux paires de vecteurs minimaux, et peut donc servir d'ancrage pour l'algorithme. Celui-ci étant exhaustif, le graphe de contiguïté est linéaire :

$$
\ldots Q(-2) \leftrightarrow Q(-1) \leftrightarrow Q(0) \leftrightarrow Q(1) \leftrightarrow Q(2) \ldots
$$

Par ailleurs, l'espace $T$ est fermé pour le produit matriciel, et la rotation de $\pi / 2$ dans le plan fournit une $T$-équivalence entre $Q(j)$ et $Q(-j)$. Une forme $T$-parfaite a 2 , ou parfois 3 paires de vecteurs minimaux, donnés comme dans le cas général à l'aide de fractions de Farey. La classification des formes $T$-parfaites en fonction de $\phi$ est donnée par le 
THÉORÈmE. (a) Si $\phi$ est rationnel, le graphe de Voronoï est fini.

(b) Si $\phi$ est irrationnel et $\kappa$ rationnel, le graphe est infini mais périodique: il existe une translation du graphe qui est une T-équivalence.

(c) Si $\kappa$ est irrationnel, deux formes $Q( \pm i)$ et $Q( \pm j)$ avec $i \neq \pm j$ ne sont jamais $T$-équivalentes, et le graphe est infini. Il existe donc une infinité de classes de T-équivalence.

Démonstration. (a) Si $\phi$ est rationnel, la droite de pente $\phi$ contient un point à coordonnées entières. Il existe alors une ellipse $T$-parfaite avec l'extrémité d'un de ses axes à coordonnées entières. L'algorithme rencontre une impasse, et la liste des formes $T$-parfaites est finie.

(b) Il n'y a pas d'impasse pour l'algorithme puisque $\phi$ est irrationnel. Posons $\kappa=\alpha / \beta$ et $D=\alpha^{2}+4 \beta^{2}$. Prenons ensuite $\left(u_{0}, v_{0}\right)$ une solution primitive de l'équation de Pell $u^{2}-D v^{2}=1$. Alors un calcul facile montre que la matrice

$$
U=\left(\begin{array}{cc}
u_{0}+\alpha v_{0} & -2 \beta v_{0} \\
-2 \beta v_{0} & u_{0}-\alpha v_{0}
\end{array}\right)
$$

appartient à $T$. Mais d'autre part, $U$ appartient à $G l(2, \mathbb{Z})$ et par conséquent $U^{2}$ est $T$-équivalente à la matrice-unité! Comme une $T$-équivalence conserve les relations de contiguïté, le graphe est bien périodique.

(c) Si $\kappa$ est irrationnel, aucune forme, à part $Q(0)$, n'est à la fois $T$ parfaite et rationnelle. Les seules $T$-équivalences possibles sont les éléments de $G l(2, \mathbb{Z})$ qui fixent la matrice-unité, en d'autres termes les matrices orthogonales. Comme celles-ci sont en nombre fini, il existe une infinité de formes $T$-inéquivalentes.

\section{Exemples et commentaires}

(a) $\phi=5 / 3=[1,1,2]$. On obtient successivement les matrices :

- $Q(0)=\left(\begin{array}{ll}1 & 0 \\ 0 & 1\end{array}\right)$, minimaux $\left(\begin{array}{l}1 \\ 0\end{array}\right)$ et $\left(\begin{array}{l}0 \\ 1\end{array}\right)$, déterminant 1 .

- $Q(1)=\left(\begin{array}{cc}15 / 7 & -15 / 14 \\ -15 / 14 & 1\end{array}\right)$, minimaux $\left(\begin{array}{l}0 \\ 1\end{array}\right)$ et $\left(\begin{array}{l}1 \\ 1\end{array}\right)$, déterminant 195/196.

- $Q(2)=\left(\begin{array}{cc}13 / 3 & -5 / 2 \\ -5 / 2 & 5 / 3\end{array}\right)$, minimaux $\left(\begin{array}{l}1 \\ 1\end{array}\right)$ et $\left(\begin{array}{l}1 \\ 2\end{array}\right)$, déterminant $35 / 36$.

- $Q(3)=\left(\begin{array}{cc}363 / 17 & -435 / 34 \\ -435 / 34 & 131 / 17\end{array}\right)$, minimaux $\left(\begin{array}{l}1 \\ 2\end{array}\right)$ et $\left(\begin{array}{l}3 \\ 5\end{array}\right)$, déterminant 987/1156.

L'algorithme s'arrête, puisque le grand axe de l'ellipse est un vecteur minimal. Il est intéressant de constater que les segments successifs du développement de $5 / 3$ en fraction continue sont donnés (avec les conventions usuelles) par la suite

$$
\text { (0/1), (1/0), (1/1), (2/1), (5/3). }
$$

(b1) $\phi=(\sqrt{13}+2) / 3=[1,1,6,1,1,1,1,6,1,1, \ldots]$. Alors $\kappa=4 / 3$. On obtient successivement les matrices : 
- $Q(0)=\left(\begin{array}{ll}1 & 0 \\ 0 & 1\end{array}\right)$, minimaux $\left(\begin{array}{l}1 \\ 0\end{array}\right)$ et $\left(\begin{array}{l}0 \\ 1\end{array}\right)$, déterminant 1 .

- $Q(1)=\left(\begin{array}{cc}3 & -3 / 2 \\ -3 / 2 & 1\end{array}\right)$, minimaux $\left(\begin{array}{l}0 \\ 1\end{array}\right),\left(\begin{array}{l}1 \\ 1\end{array}\right),\left(\begin{array}{l}1 \\ 2\end{array}\right)$, déterminant $3 / 4$.

- $Q(2)=\left(\begin{array}{cc}199 & -213 / 2 \\ -213 / 2 & 57\end{array}\right)$, minimaux $\left(\begin{array}{l}1 \\ 2\end{array}\right),\left(\begin{array}{c}7 \\ 13\end{array}\right),\left(\begin{array}{c}8 \\ 15\end{array}\right)$, déterminant $3 / 4$.

- $Q(3)=\left(\begin{array}{cc}1009 & -540 \\ -540 & 289\end{array}\right)$, minimaux $\left(\begin{array}{c}8 \\ 15\end{array}\right)$ et $\left(\begin{array}{c}15 \\ 28\end{array}\right)$, déterminant 1 .

Cet exemple contient la forme parfaite ayant 3 paires de vecteurs minimaux, et sa période est courte grâce à l'existence d'une solution de l'équation de Pell $u^{2}-13 v^{2}=-1$. La périodicité est donnée par l'équation matricielle

$$
\left(\begin{array}{cc}
1009 & -540 \\
-540 & 289
\end{array}\right)=\left(\begin{array}{cc}
28 & -15 \\
-15 & 8
\end{array}\right)\left(\begin{array}{ll}
1 & 0 \\
0 & 1
\end{array}\right)\left(\begin{array}{cc}
28 & -15 \\
-15 & 8
\end{array}\right) \text {. }
$$

Par ailleurs, les segments successifs du développement de $\phi$ en fraction continue sont

$$
(0 / 1),(1 / 0),(1 / 1),(2 / 1),(13 / 7),(15 / 8),(28 / 15),(43 / 23), \ldots
$$

(b2) $\phi=(\sqrt{97}+4) / 9$. Alors $\kappa=8 / 9$. Cet exemple est très semblable au précédent, mais il met en évidence une curieuse anomalie : les approximations de $\phi$ n'apparaissent pas toutes comme coordonnées des vecteurs minimaux. Il y manque par exemple $(17 / 11)$ et $(377 / 245)$. La donnée de $\phi$ ne permet donc pas de prédire quels sont les vecteurs minimaux des formes $Q(k)$.

(c) $\phi=\sqrt{2}=[1,2,2,2,2, \ldots]$. Comme prévu par le théorème, la forme $Q(k)$ n'est $T$-équivalente qu'à elle-même et à $Q(-k)$. Vu l'absence de 1's dans le développement de $\sqrt{2}$, toutes les paires consécutives d'approximations apparaissent comme vecteurs minimaux des formes. Par calcul direct, on constate que tous les déterminants des formes $Q( \pm k)$ sont différents. La liste des formes $T$-parfaites est donc également infinie si l'on quotiente par l'action de $G l(2, \mathbb{Z})$.

\section{Bibliographie}

[BMS] A.-M. Bergé, J. Martinet et F. Sigrist, Une généralisation de l'algorithme de Voronoï pour les formes quadratiques, Astérisque 209 (1992), 137-158.

[Ja1] D.-O. Jaquet-Chiffelle, Enumération complète des classes de formes parfaites en dimension 7, Ann. Inst. Fourier (Grenoble) 43 (1) (1993), 21-55.

[Ja2] -, Trois théorèmes de finitude pour les G-réseaux, en préparation.

[Vo] G. Voronoï, Sur quelques propriétés des formes quadratiques positives parfaites, J. Reine Angew. Math. 133 (1908), 97-178.

INSTITUT DE MATHÉMATIQUES UNIVERSITÉ DE NEUCHÂTEL RUE EMILE ARGAND 11 CH-2007 NEUCHÂTEL, SUISSE 